\title{
Joint effects of prenatal exposure to per- and poly-fluoroalkyl substances and psychosocial stressors on corticotropin-releasing hormone during pregnancy
}

\author{
Stephanie M. Eick $\mathbb{D}^{1} \cdot$ Dana E. Goin ${ }^{1} \cdot$ Lara Cushing $^{2} \cdot$ Erin DeMicco $^{1} \cdot$ Sabrina Smith $^{3} \cdot$ June-Soo Park $^{3}$. \\ Amy M. Padula ${ }^{1} \cdot$ Tracey J. Woodruff $^{1} \cdot$ Rachel Morello-Frosch $^{1,4}$
}

Received: 3 December 2020 / Revised: 26 February 2021 / Accepted: 18 March 2021 / Published online: 6 April 2021

(c) The Author(s) 2021. This article is published with open access

\begin{abstract}
Background Prenatal exposure to per- and poly-fluoroalkyl substances (PFAS) and psychosocial stressors has been associated with adverse pregnancy outcomes, including preterm birth. Previous studies have suggested that joint exposure to environmental chemical and social stressors may be contributing to disparities observed in preterm birth. Elevated corticotropin-releasing hormone $(\mathrm{CRH})$ during mid-gestation may represent one biologic mechanism linking chemical and nonchemical stress exposures to preterm birth.

Methods Using data from a prospective birth cohort $(N=497)$, we examined the cross-sectional associations between five individual PFAS (ng/mL; PFNA, PFOA, PFOS, PFHxS, and Me-PFOSA-AcOH) and CRH (pg/mL) using linear regression. PFAS and CRH were measured during the second trimester in serum and plasma, respectively. Coefficients were standardized to reflect change in CRH associated with an interquartile range (IQR) increase in natural log-transformed PFAS. We additionally examined if the relationship between PFAS and CRH was modified by psychosocial stress using stratified models. Self-reported depression, stressful life events, perceived stress, food insecurity, and financial strain were assessed using validated questionnaires during the second trimester and included as binary indicators of psychosocial stress.

Results An IQR increase in PFNA was associated with elevated CRH $(\beta=5.17,95 \%$ confidence interval $[\mathrm{CI}]=1.79,8.55)$. Increased concentrations of PFOA were also moderately associated with $\mathrm{CRH}(\beta=3.62,95 \% \mathrm{CI}=-0.42,7.66)$. The relationship between PFNA and CRH was stronger among women who experienced stressful life events, depression, food insecurity, and financial strain compared to women who did not experience these stressors.

Conclusions This cross-sectional study is the first to examine the relationship between PFAS exposure and CRH levels in mid-gestation. We found that these associations were stronger among women who experienced stress, which aligns with previous findings that chemical and nonchemical stressor exposures can have joint effects on health outcomes.
\end{abstract}

Keywords Per- and poly-fluoroalkyl substances $\cdot$ Stress $\cdot$ Pregnancy $\cdot$ Health disparities

Supplementary information The online version contains supplementary material available at https://doi.org/10.1038/s41370021-00322-8.

Stephanie M. Eick

stephanie.eick@ucsf.edu

Rachel Morello-Frosch

rmf@berkeley.edu

1 Program on Reproductive Health and the Environment, Department of Obstetrics, Gynecology and Reproductive Sciences, University of California, San Francisco, San Francisco, CA, USA

\section{Introduction}

Exposure to per- and poly-fluoroalkyl substances (PFAS) is ubiquitous in the US, with $>95 \%$ of individuals having

2 Department of Environmental Health Sciences, Fielding School of Public Health, University of California, Los Angeles, Los Angeles, CA, USA

3 Environmental Chemistry Laboratory, Department of Toxic Substances Control, California Environmental Protection Agency, Berkeley, CA, USA

4 Department of Environmental Science, Policy and Management and School of Public Health, University of California, Berkeley, Berkeley, CA, USA 
detectable serum levels [1]. PFAS are endocrine disrupting chemicals (EDCs) that are highly persistent in the environment and prevalent in a wide range of consumer products including cookware, clothing, and food packaging, as well as food and drinking water sources [2-4]. More than 4000 PFAS have been identified and levels of some PFAS bioaccumulate in humans [2]. Previous studies have linked prenatal PFAS exposure to increased risk of adverse pregnancy outcomes. For example, increased concentrations of perfluorooctanoic acid (PFOA), perflucorooctane sulfonic acid (PFOS), and perfluorononanoic acid (PFNA) have been associated with increased odds of preterm birth (defined as gestational age at birth $<37$ weeks) and a reduction in gestational age at birth $[5,6]$. Other studies have shown that PFAS chemicals are easily transmitted to the developing fetus via the placenta [7] and present in the cord blood of newborns [8]. Despite the consistency in these associations, the biologic pathways that link PFAS to preterm birth largely remain unknown.

Experiences of psychosocial stressors during pregnancy, including stressful life events, poor neighborhood quality, and responses to such stressors, such as depression and anxiety, have also been associated with preterm birth [912]. Furthermore, there may be a cumulative or joint effect of chemical and psychosocial stressors [13]. This may be especially relevant for lower socioeconomic status (SES) individuals, who are often exposed to multiple environmental and social stressors simultaneously [14]. It is possible that these chemical and nonchemical stressors affect similar biologic mechanisms and thus may lead to adverse outcomes through similar pathways.

The hypothalamic pituitary adrenal (HPA) axis has emerged as one mechanism linking prenatal stress exposure to preterm birth [15]. It is hypothesized that experiencing psychosocial stress activates the maternal HPA axis, increasing cortisol production, which is followed by production of corticotropin-releasing hormone (CRH). Since the placenta also produces CRH and CRH levels during pregnancy are considered part of a "biological clock" regulating the length of gestation [16, 17], early excess CRH production may result in preterm birth [15]. This pathway has been examined in animal models [18] and human studies have shown that certain psychosocial stressors are associated with elevated CRH during the second and third trimesters of pregnancy [19-22].

To date, no studies have examined the association between prenatal PFAS exposure and CRH during pregnancy. However, increasing concentrations of other classes of EDCs, including phenols, parabens, and phthalates, have been associated with alternations in HPA axis function in animal models [23] and during human pregnancy [24]. For example, increases in CRH levels between 24 and 28 weeks gestation have been observed in association with increasing 2,4-dichlorophenol and triclosan levels [25]. In contrast, inverse associations between phthalate metabolites and CRH, measured at 16-20 and 24-28 weeks gestation, were detected in a pregnancy cohort in Puerto Rico [26]. Elevated levels of phthalates and phenols have also been linked to an increased risk of preterm birth [27, 28]. Previous work has identified a joint effect of stressful life events and EDCs with respect to gestational length at delivery. In these studies, inverse associations between certain phenol and phthalate metabolites and gestational age were stronger among women who experienced stressful events [29, 30].

In the present study, we utilized data from a demographically diverse group of pregnant women in San Francisco, CA to assess the relationship between PFAS exposures and $\mathrm{CRH}$ during pregnancy. We hypothesized that increasing PFAS exposure would be associated with elevated CRH during the second trimester and that these associations would be stronger among women who experienced psychosocial stress.

\section{Methods}

\section{Study population}

This study utilized data from 497 participants enrolled in the Chemicals in Our Bodies (CIOB) cohort, which has been previously described in detail [31]. Briefly, CIOB was designed to examine the cumulative effects of environmental chemicals and psychosocial stressors on fetal growth and offspring development. Women were recruited during their second trimester from three University of California, San Francisco hospitals between 2014 and 2018. Participants recruited from the Zuckerberg San Francisco General Hospital were primarily lower income and enrolled in MediCal (California's Medicaid program), whereas women recruited from Moffitt Long and Mission Bay Hospitals were economically diverse, and the majority had private health insurance. Women were eligible for enrollment in CIOB if they were $\geq 18$ years of age, not pregnant with multiples, and spoke English or Spanish as their primary language. As part of the study, mothers consented to study staff accessing their medical records. The Institutional Review Boards at the University of California, San Francisco (10-00861) and Berkeley (2010-05-04) approved CIOB and all participants provided written, informed consent prior to participating. All study personnel were blinded to participants' CRH and PFAS levels.

\section{Per- and poly-fluoroalkyl substances (PFAS)}

Maternal serum samples were collected during the second trimester between 12 and 28 weeks gestation in red top tubes 
and stored at $-80{ }^{\circ} \mathrm{C}$ until analysis for 12 PFAS. Analyses were performed by the Environmental Chemical Laboratory at the California Department of Toxic Substances Control. Method detection limits (MDL) were calculated as three times the standard deviation (SD) of the blank concentrations for all PFAS. PFAS were analyzed by injection onto an automated on-line solid phase extraction method coupled to liquid chromatography and tandem mass spectrometry. Additional details regarding PFAS measurement and quality assurance and quality control is provided elsewhere $[8,32]$. We focused our analysis on those PFAS chemicals with $>80 \%$ detection, which included PFNA, PFOS acid, PFOA, methylperfluorooctane sulfonamide acetic acid (Me-PFOSA$\mathrm{AcOH})$, and perfluorohexanesulphonic acid (PFHxS). This cut point was utilized for consistency with our prior work in this cohort [32]. While other PFAS compounds had machineread values $>80 \%$, variability in PFAS levels across our population for these compounds was limited. In our analysis, measurements below the MDL were assigned the machineread value if a signal was detected ( $<2 \%$ of observations). If no signal was detected, measurements below the MDL were treated as missing $(<0.5 \%$ of observations). PFAS $(\mathrm{ng} / \mathrm{mL})$ concentrations were right-skewed and natural log-transformed for analysis.

\section{Corticotropin-releasing hormone (CRH)}

Blood samples were collected during the second trimester (mean 20.7 weeks, range 12-28 weeks) and were centrifuged to extract plasma. Plasma samples were subsequently aliquoted and frozen at $-80{ }^{\circ} \mathrm{C}$ until analysis. Analysis for CRH levels ( $\mathrm{pg} / \mathrm{mL}$ ) was conducted by the Fisher Lab at the University of California, San Francisco using enzyme immunoassay kits (Phoenix Pharmaceutical, Burlingame, CA) and standard operating protocols. Samples were acidified and loaded onto pretreated SEP-COLUMNs, and resultant eluent was evaporated and redissolved in RIA buffer for radioimmunoassay. If subsequent concentrations were not within range of detection, samples were diluted or reconcentrated accordingly.

\section{Psychosocial stress}

Psychosocial stressors and responses to stress were assessed via an interview questionnaire administered by study personnel during a second trimester prenatal care visit. Psychosocial stressors included stressful life events [33, 34], financial strain [35], and food insecurity [36, 37]. Perceived stress [38] and depression [39] were included as measures of stress response. A detailed description of the categorization of psychosocial stressors and stress response measures is available elsewhere [40] and is summarized below.

\section{Stressful life events}

Women were considered to have experienced stressful life events if they reported experiencing two or more of the following events within the last 12 months: a close family member was hospitalized, separation or divorce from her partner, she or her partner lost their job, she moved to a new address, a close family member experienced immigration problems, she argued with her partner more than usual, her partner did not want her to be pregnant, she was in a physical fight, she could not pay her bills, her partner had legal trouble, someone close to her was drinking or using drugs, or someone close to her passed away $[33,34]$.

\section{Financial strain}

Women were classified as experiencing financial strain if their annual household income was below the 2017 San Francisco county poverty line or they reported finding it difficult to pay for basic necessities, such as food, housing, medical care, or utilities [35].

\section{Food insecurity}

If women reported that within the past 12 months they had skipped meals, ate less than they should, or were hungry but did not eat because there was not enough money for food, they were classified as being food insecure. Women were also considered to be food insecure if they reported that the food they bought did not last or if there was not enough money for more food or if they could not afford to eat balanced meals [36, 37].

\section{Perceived stress}

Perceived stress was assessed using the Perceived Stress Scale-4 (PSS-4) $[38,41]$. The range of scores on the PSS-4 was $0-13$. For consistency with prior work, the standardized cut point of $\geq 9$ was used to denote experiences of moderate or severe perceived stress [42, 43].

\section{Depression}

The 10-item Center for Epidemiologic Studies-Depression (CES-D) was used to measure depression [39]. CES-D scores ranged from 0 to 30 , and the clinical cut point for depressive symptoms $(\geq 16)$ was used to classify participants as having experienced depression [44].

\section{Demographics}

Maternal age at enrollment, pre-pregnancy body mass index $\left(\mathrm{BMI} ; \mathrm{kg} / \mathrm{m}^{2}\right)$, parity, gestational age at study visit and 
gestational age at delivery were obtained from the mother's abstracted medical record. On the medical record, gestational age is calculated using the clinician's best estimation of chronological gestational age based on last menstrual period, early ultrasound, and/or in vitro fertilization date. Maternal race/ethnicity (Non-Hispanic (NH) White, NHBlack, NH-Asian/Pacific Islander, NH-Other/Multi-Racial and Latina), marital status (married, living with a partner, single), and maternal educational attainment (less than high school, high school or some college, college degree, graduate degree) were obtained via self-report at the second trimester interview.

\section{Statistical analysis}

We examined the distribution of $\mathrm{CRH}$ and PFAS across demographic characteristics and psychosocial stressors using geometric means and geometric SD. Loess curves were used to assess the relationship between $\mathrm{CRH}$ and individual PFAS, and no evidence of nonlinearity was observed. Thus, linear regression models were used to estimate unadjusted and adjusted associations between PFAS, psychosocial stressors, and CRH. Coefficients were standardized to reflect the change in $\mathrm{CRH}$ associated with an interquartile range (IQR) increase in PFAS levels for ease of interpretation. An IQR increased was defined by the difference between the 75 th and 25 th percentiles. QQ plots were examined to ensure that regression residuals were normally distributed. We a priori controlled for gestational age at study visit in all models (unadjusted and adjusted), as CRH increases throughout pregnancy and we previously observed elevated PFAS and preterm birth odds ratios in this study population [32]. Maternal age, race/ethnicity, education, and parity were included as additional covariates in final adjusted models because they were associated with $\mathrm{CRH}$, stress, and PFAS in bivariate analyses, and previous literature suggests an association with our exposures and outcome [45, 46]. We additionally adjusted for pre-pregnancy BMI and nativity as a sensitivity analysis and point estimates did not change substantially. A complete case analysis was used for all models.

We examined psychosocial stressors, including stressful life events, perceived stress, depression, food insecurity, and financial strain, as potential effect modifiers of the relationship between PFAS and CRH. In these analyses, we estimated the joint effects of PFAS and psychosocial stressors by examining the additive association between individual PFAS and CRH stratified by levels of each binary stressor, adjusting for gestational age at sample collection, maternal age, race/ethnicity, education, and parity. Separate models included $p$ values for the interaction terms between PFAS and binary psychosocial stressors, which we used to assess additive interaction at $p<0.10$. All statistical analyses were conducted in R Version 4.0.1.

\section{Results}

The majority of women in the CIOB study population were $>30$ years of age $(73 \%)$, married $(66 \%)$, and had a college $(23 \%)$ or graduate education $(36 \%)$. More than half of women $(57 \%)$ experienced at least two stressful life events within the past year and $34 \%$ experienced financial strain (Table 1). The geometric mean of CRH was higher among White women compared to Latina women (33.02 versus $25.28 \mathrm{pg} / \mathrm{mL}$ ). Women who were classified as experiencing financial strain and depression also had elevated CRH levels relative to women who did not report these stressors (Table 1). Perceived stress, depression, financial strain, food insecurity, and stressful life events were moderately to strongly correlated with one another [40].

The five PFAS that were the focus of our analysis were detected in over $98 \%$ of women. The distribution of PFAS varied widely across compounds (Table 2). The median level was highest for PFOS (1.92 ng/mL) and PFOA (0.76 $\mathrm{ng} / \mathrm{mL})$, and lowest for Me-PFOSA-AcOH $(0.05 \mathrm{ng} / \mathrm{mL})$. The distribution of PFAS with $<80 \%$ detection is shown in Table S1. The median level of CRH was $32.56 \mathrm{pg} / \mathrm{mL}$.

In our study population, PFAS levels were generally higher among women who were older, married, and had at least a college education (Table S2). With the exception of PFOS, levels of all PFAS were lower among Latina and Black women relative to other racial and ethnic groups. Levels of PFNA, PFOA, and PFOS were lower among women who experienced food insecurity and financial strain (Table S2).

In unadjusted models adjusted only for gestational age at visit, we observed an increase in CRH $(\mathrm{pg} / \mathrm{mL})$ per unit IQR increase in PFNA $(\beta=6.26,95 \%$ confidence interval $[\mathrm{CI}]=3.31,9.21)$, PFOA $(\beta=5.11,95 \% \mathrm{CI}=1.70,8.52)$, and PFOS $(\beta=4.38,95 \% \mathrm{CI}=1.06,7.70)$. Only the PFNA effect remained relatively unchanged after adjustment for the additional covariates $(\beta=5.17,95 \% \mathrm{CI}=1.79,8.55)$ (Fig. 1 and Table S3). In adjusted models, a high level of perceived stress was associated with higher $\mathrm{CRH}$ in $\mathrm{pg} / \mathrm{mL}$ compared to women who did not experience perceived stress $(\beta=10.2,95 \% \quad \mathrm{CI}=1.82,18.57)$. Depression, stressful life events, food insecurity, and financial strain were associated with $\mathrm{CRH}$ in the direction hypothesized after adjustment, however the confidence intervals included the null value (Fig. 1 and Table S4).

In adjusted linear regression models stratified by each stressor, we found that the relationship between PFNA and 
Table 1 Description of Chemicals in Our Bodies study population and geometric mean (geometric standard deviation) of corticotropinreleasing hormone $(\mathrm{pg} / \mathrm{mL})$ across demographic characteristics.

\begin{tabular}{ll}
\hline$N(\%)$ & $\begin{array}{l}\text { CRH geometric mean } \\
\text { (geometric SD) }\end{array}$ \\
\hline
\end{tabular}

Maternal age, years

$18-24$

25-29

30-34

$\geq 35$

Missing

Maternal education

Less than high school

High school degree or some college

College degree

Graduate degree

Missing

Maternal race/ethnicity

White

Black

Asian/Pacific Islander

Latina

Other/Multi-Racial

Missing

Pre-pregnancy body mass index

Underweight $\left(<18.5 \mathrm{~kg} / \mathrm{m}^{2}\right)$

Normal $\left(18.5-24.9 \mathrm{~kg} / \mathrm{m}^{2}\right)$

Overweight $\left(25-29.9 \mathrm{~kg} / \mathrm{m}^{2}\right)$

Obese $\left(\geq 30 \mathrm{~kg} / \mathrm{m}^{2}\right)$

Missing

Parity

No prior births

One or more prior births

Missing

Marital status

Married

Living together

Single

Missing

Perceived stress

Yes

No

Missing

Depression

Yes

No

Missing

Stressful life events

Yes

No

Missing

Financial strain

Yes

No

Missing

Food insecurity

Yes

No

Missing

$N=497$.

$S D$ standard deviation.
CRH was stronger among women who experienced stressful life events $(\beta=7.06,95 \% \mathrm{CI}=2.82,11.3)$, depression $(\beta=20.77,95 \% \mathrm{CI}=1.56,39.99)$, food insecurity $(\beta=$ 9.52, 95\% $\mathrm{CI}=1.72,17.32)$, and financial strain $(\beta=$ $10.61,95 \% \mathrm{CI}=4.90,16.33)$ compared to women who did not experience these stressors (Fig. 2 and Tables S5, S7S9). Among women who did not experience perceived stress, an IQR increase in PFOA was associated with a $4.61 \mathrm{pg} / \mathrm{mL}$ increase in $\mathrm{CRH}(95 \% \mathrm{CI}=0.28,8.95)$ (Fig. 2 and Table S6). Associations between PFOS, PFHxS, and $\mathrm{CRH}$ were stronger among women who were food insecure relative to those who did not report food insecurity (Fig. 2 and Table S8). In contrast, a modest and imprecise inverse association was observed between PFOS $(\beta=-3.88,95 \%$ $\mathrm{CI}=-27.33,19.56), \quad \mathrm{PFHxS} \quad(\beta=-10.23,95 \% \quad \mathrm{CI}=$ $-41.98,21.52)$, Me-PFOSA-AcOH $\quad(\beta=-10.12,95 \%$ $\mathrm{CI}=-29.15,8.91)$, and CRH among women who experienced depression. With the exception of PFNA, the relationship between PFAS and CRH among those who did not experience depression was null (Fig. 2 and Table S7). Tests for potentially interactive effects for each stressor and stress response were not significant at $p<0.1$.

\section{Discussion}

Among a diverse population of pregnant women in San Francisco, we found that an IQR increase in levels of PFNA in maternal serum was associated with higher CRH concentrations during the second trimester. The relationship between PFNA and CRH was stronger among women who experienced stressful life events, depression, food insecurity, and financial strain relative to women who did not experience these stressors but tests for interactive effects were not statistically significant. We also observed an association between perceived stress and higher prenatal $\mathrm{CRH}$. Our findings contribute to the growing body of literature supporting a joint effect of environmental chemical and psychosocial stressors on biomarkers of stress response.

In our study population, we found that women who reported high levels of perceived stress had elevated levels of CRH compared to women who experienced no or low perceived stress when assessed at the second trimester. This finding is consistent with previous research. Among 85 pregnant women in Utah, the mean score on the PSS was marginally elevated among women with $\mathrm{CRH} \geq 15 \mathrm{pcg} / \mathrm{mL}$ compared to those with $\mathrm{CRH}<15 \mathrm{pcg} / \mathrm{mL}$ (21.1 versus 18.2) when CRH was measured between 14 and 20 weeks gestation [20]. Similarly, a path analysis conducted among Hispanic pregnant women in Texas found that perceived stress was positively associated with $\mathrm{CRH}$ at 22-25 weeks gestation [47]. In contrast, in our study population, none of the other stressors examined were associated with second 
Table 2 Distribution of second trimester plasma levels of corticotropin-releasing hormone $(\mathrm{pg} / \mathrm{mL})$ and second trimester serum levels of per- and polyfluoroalkyl substances $(\mathrm{ng} / \mathrm{mL})$ $(N=497)$.

\begin{tabular}{|c|c|c|c|c|c|c|c|c|}
\hline & \multirow{2}{*}{$\begin{array}{l}\% \text { Above } \\
\text { MDL }\end{array}$} & \multirow{2}{*}{$\begin{array}{l}\text { \% Machine } \\
\text { readable }\end{array}$} & \multirow{2}{*}{$\begin{array}{l}\text { Geometric mean } \\
\text { (geometric SD) }\end{array}$} & \multicolumn{5}{|c|}{ Percentile } \\
\hline & & & & 5 th & 25 th & 50th & 75th & 95th \\
\hline $\mathrm{CRH}$ & 100.00 & 100.00 & $30.10(2.19)$ & 8.62 & 17.77 & 32.56 & 50.73 & 99.21 \\
\hline PFNA & 98.79 & 99.60 & $0.30(1.92)$ & 0.10 & 0.20 & 0.30 & 0.43 & 0.85 \\
\hline PFOA & 99.80 & 100.00 & $0.73(1.97)$ & 0.25 & 0.46 & 0.76 & 1.12 & 2.11 \\
\hline PFHxS & 100.00 & 100.00 & $0.36(2.26)$ & 0.11 & 0.20 & 0.32 & 0.58 & 1.49 \\
\hline PFOS & 100.00 & 100.00 & $1.86(2.07)$ & 0.51 & 1.18 & 1.92 & 3.11 & 6.00 \\
\hline $\begin{array}{l}\text { Me-PFOSA- } \\
\text { AcOH }\end{array}$ & 98.79 & 99.80 & $0.05(2.07)$ & 0.02 & 0.03 & 0.05 & 0.08 & 0.18 \\
\hline
\end{tabular}

$S D$ standard deviation; $M D L$ method detection limit.

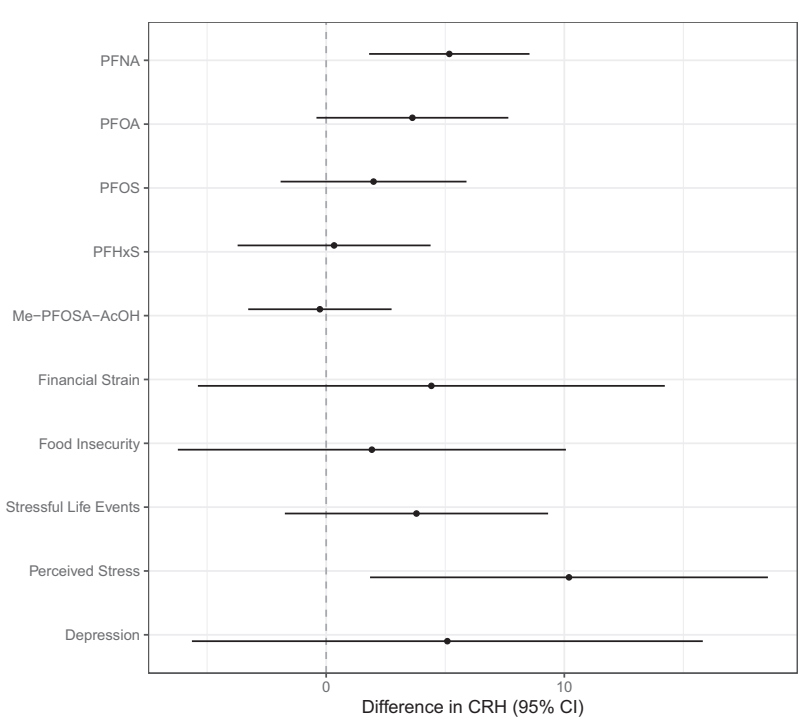

Fig. 1 Adjusted linear regression coefficients and 95\% confidence intervals for second trimester CRH concentrations $(\mathrm{pg} / \mathrm{mL})$ in maternal plasma in relation to psychosocial stressors and an interquartile range increase in second trimester PFAS concentrations $(\mathbf{n g} / \mathbf{m L})$ in maternal serum. PFAS are natural $\log$ transformed. Models adjusted for gestational age at study visit, maternal age (continuous), maternal education, maternal race/ethnicity, and parity.

trimester CRH. While these null associations are in contrast to our hypotheses and prior work [21, 22], they are consistent with prior findings. For example, among a prospective birth cohort in North Carolina, mid-pregnancy placental CRH (measured twice between 20 and 24-29 weeks gestation) was not elevated in response to prenatal depression and furthermore that study found no association between prenatal $\mathrm{CRH}$ and postnatal depression [48]. It is possible that we did not observe stronger effects between stress and $\mathrm{CRH}$ due to the timing of its measurement during pregnancy. We measured CRH during the second trimester (mean 20 weeks) and other studies have shown an association between stress and CRH generally measured CRH during the third trimester [19, 21, 22]. This may indicate that the third trimester is a more susceptible time period for psychosocial stress exposure. It is possible that $\mathrm{CRH}$ is also sensitive to more acute stress exposure. In our study population, we observed a positive association between $\mathrm{CRH}$ and perceived stress using the PSS, which asks about experiences of stress within the last month. Chronic stress exposure suppresses the HPA axis [15], which may decrease the release of CRH. This may explain why measures of chronic stress, including food insecurity, depression, financial strain, and stressful life events, were not independently associated with $\mathrm{CRH}$ in our study.

PFAS bioaccumulate across the lifespan and exposure is ubiquitous among pregnant women in the US [2]. However, PFAS levels were lower in our cohort relative to other study populations, including NHANES [31], which could be due to some PFAS levels declining over time as a result of voluntary industry phase-outs of PFOA and PFOS. We also observed that PFAS levels were higher among those with higher educational attainment, which could be due to SESrelated differences in product use and consumption of certain foods known to contain PFAS, including fish. PFAS are also excreted via breastmilk [2], which may explain why PFAS levels were lower among multiparous women in our study. These factors may be driving differences we observed in adjusted versus unadjusted models. Latina participants in our cohort also had lower PFAS levels relative to other racial and ethnic groups, which could be attributed to the high percentage of Latinas in our study born outside of the US and is consistent with past work [49].

Our study is the first to examine $\mathrm{CRH}$ as a potential physiologic response to PFAS exposure during pregnancy. Higher levels of CRH may represent one possible biologic pathway linking PFAS and stress exposures to preterm birth, shortened gestational length, fetal growth restriction, and early childhood neurodevelopment $[50,51]$. Prior work has shown that levels of PFOA and PFOS decrease moderately from early pregnancy to birth [52], possibly as a 

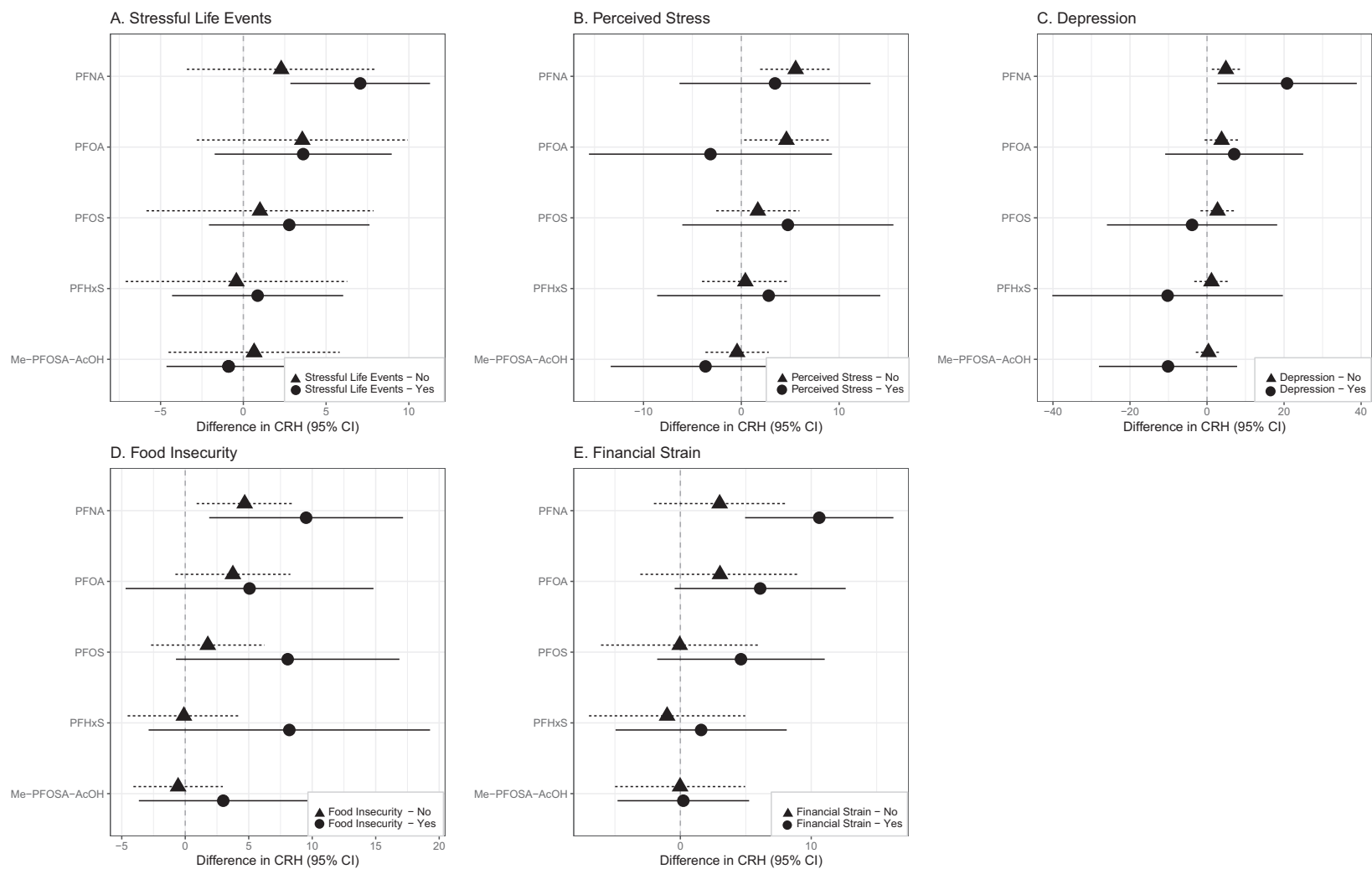

Fig. 2 Adjusted linear regression coefficients and 95\% confidence intervals for second trimester CRH concentrations $(\mathrm{pg} / \mathrm{mL})$ in maternal plasma with an interquartile range increase in second trimester PFAS concentrations $(\mathrm{ng} / \mathrm{mL})$ in maternal serum stratified by binary indicators of psychosocial stress. PFAS are natural log-transformed. Models adjusted for gestational age at study visit, maternal age (continuous), maternal education, maternal race/ ethnicity, and parity. result of transplacental transfer of PFAS. CRH, measured longitudinally, increases over the course of pregnancy and elevated CRH during early gestation (16-20 weeks) is associated with a marked increase in the risk of preterm birth [15]. Previously in the CIOB cohort, compared to women in the lowest tertile of PFNA exposure, women in the upper two tertiles had $1.88(95 \% \mathrm{CI}=0.83,4.30)$ and $2.06(95 \% \mathrm{CI}=0.72,5.89)$, respectively, increased odds of preterm birth [32]. While this finding was not statistically significant, the magnitude and direction of these effects suggest a potential dose-response relationship. Of all PFAS examined in relation to preterm birth in our previous work, PFNA had one of the strongest effects [32], which is consistent with our observations in this study with respect to PFNA and CRH levels.

Our prior work in the CIOB cohort has also shown that many of the stressors examined here have a joint effect on infant birthweight for gestational age [53]. CRH plays a critical role in regulating the physiologic stress response. Under the experiences of acute stress, the HPA axis is activated, leading to the release of adrenocorticotropic hormone, which signals the release of cortisol [15]. High cortisol levels signal to the hypothalamus to release CRH.
Chronic activation of the HPA axis may lead to dysregulation of the neuroimmunological and neurohormonal systems, and may result in excess CRH and cortisol in maternal circulation [15]. This pathway may also be altered by endocrine disruption, as previous work has shown that pregnant women with high mercury and stress levels have a blunted morning cortisol response relative to women with lower mercury levels [54]. Elevated prenatal CRH levels have also been observed following exposure to some phthalate and phenol metabolites $[25,26]$. The upstream biomarkers of physiologic effect associated with environmental chemical and social stressors deserve further exploration in future studies.

Our study has many important strengths. We included multiple measures of stress and responses to stress and our study population included a demographically diverse group of pregnant women. We also acknowledge our limitations. Our study population was not a random sample and thus findings may not be generalizable to other pregnant populations. PFAS, stress, and CRH were measured at the same time point, which raises temporality challenges typical of cross-sectional study designs. However, it is unlikely that CRH or stress would affect PFAS levels. Furthermore, our 
sample size was modest and this imprecision is reflected in the wide confidence intervals observed for some of our point estimates. In addition, we dichotomized all psychosocial stress measures in our analyses. While we did use previously establish cut points for all scales, this could have resulted in a loss of statistical power. Our stressful life events scale also did not capture the subjective assessment of stressful events, which may introduce misclassification depending on the perception of these events. Further, we did not make adjustments for multiple comparisons. Although adjustment for multiple comparisons is not always necessary [55] and we focused consistent trends rather than $p$ values in interpreting our results, this may increase the possibility of chance findings. While an important strength of our study is that we examined the joint effects of chemical and nonchemical stressors, we did not examine the cumulative effects of these exposures. Last, cortisol levels and experiencing traumatic events during early pregnancy have been linked to increased risk of early fetal loss [56, 57] and as a result, our study findings may be subject to live birth bias [58].

\section{Conclusions}

Our cross-sectional study is the first to examine the effects of PFAS exposure on CRH during pregnancy and our findings highlight a stress response biomarker of potential relevance for adverse pregnancy outcomes including preterm birth. We found modestly stronger associations among women who experienced maternal stress. Future studies should focus on the potential modifying effect of stress and its potential to increase susceptibility to the effects of environmental chemicals among pregnant women.

Acknowledgements We would like to thank our clinical research team for collecting the data, the study participants who participated in the CIOB study, and the DTSC biomonitoring team for the laboratory analysis of PFAS in serum.

Funding This work was supported by grants RD83543301 from the United States Environmental Protection Agency, and P01ES022841 from the National Institute of Environmental Health Sciences, and UG3OD023272 and UH3OD023272 from the National Institutes of Health Environmental influences on Child Health Outcomes (ECHO) program. Funding for LC was also provided by the JPB Environmental Health Fellowship.

\section{Compliance with ethical standards}

Conflict of interest The authors declare no competing interests.

Publisher's note Springer Nature remains neutral with regard to jurisdictional claims in published maps and institutional affiliations.
Open Access This article is licensed under a Creative Commons Attribution 4.0 International License, which permits use, sharing, adaptation, distribution and reproduction in any medium or format, as long as you give appropriate credit to the original author(s) and the source, provide a link to the Creative Commons license, and indicate if changes were made. The images or other third party material in this article are included in the article's Creative Commons license, unless indicated otherwise in a credit line to the material. If material is not included in the article's Creative Commons license and your intended use is not permitted by statutory regulation or exceeds the permitted use, you will need to obtain permission directly from the copyright holder. To view a copy of this license, visit http://creativecommons. org/licenses/by/4.0/.

\section{References}

1. Calafat AM, Wong LY, Kuklenyik Z, Reidy JA, Needham LL. Polyfluoroalkyl chemicals in the U.S. population: data from the National Health and Nutrition Examination Survey (NHANES) 2003-2004 and comparisons with NHANES 1999-2000. Environ Health Perspect. 2007;115:1596-602.

2. Sunderland EM, Hu XC, Dassuncao C, Tokranov AK, Wagner CC, Allen JG. A review of the pathways of human exposure to poly- and perfluoroalkyl substances (PFASs) and present understanding of health effects. J Expo Sci Environ Epidemiol. 2019;29:131-47.

3. Hu XC, Andrews DQ, Lindstrom AB, Bruton TA, Schaider LA, Grandjean P, et al. Detection of poly- and perfluoroalkyl substances (PFASs) in U.S. drinking water linked to industrial sites, military fire training areas, and wastewater treatment plants. Environ Sci Technol Lett. 2016;3:344-50.

4. Domingo JL, Nadal M. Per- and polyfluoroalkyl substances (PFASs) in food and human dietary intake: a review of the recent scientific literature. J Agric Food Chem. 2017;65:533-43.

5. Meng Q, Inoue K, Ritz B, Olsen J, Liew Z. Prenatal exposure to perfluoroalkyl substances and birth outcomes; an updated analysis from the Danish National Birth Cohort. Int J Environ Res Public Health. 2018;15:1832.

6. Sagiv SK, Rifas-Shiman SL, Fleisch AF, Webster TF, Calafat $\mathrm{AM}, \mathrm{Ye} \mathrm{X}$, et al. Early-pregnancy plasma concentrations of perfluoroalkyl substances and birth outcomes in project viva: confounded by pregnancy hemodynamics? Am J Epidemiol. 2018;187:793-802.

7. Cai D, Li Q-Q, Chu C, Wang S-Z, Tang Y-T, Appleton AA, et al. High trans-placental transfer of perfluoroalkyl substances alternatives in the matched maternal-cord blood serum: evidence from a birth cohort study. Sci Total Environ. 2020;705:135885.

8. Morello-Frosch R, Cushing LJ, Jesdale BM, Schwartz JM, Guo W, Guo T, et al. Environmental chemicals in an urban population of pregnant women and their newborns from San Francisco. Environ Sci Technol. 2016;50:12464-72.

9. Ncube CN, Enquobahrie DA, Albert SM, Herrick AL, Burke JG. Association of neighborhood context with offspring risk of preterm birth and low birthweight: a systematic review and metaanalysis of population-based studies. Soc Sci Med. 2016;153:156-64.

10. Dole N, Savitz DA, Hertz-Picciotto I, Siega-Riz AM, McMahon MJ, Buekens P. Maternal stress and preterm birth. Am J Epidemiol. 2003;157:14-24.

11. Liu C, Cnattingius S, Bergström M, Östberg V, Hjern A. Prenatal parental depression and preterm birth: a national cohort study. BJOG. 2016;123:1973-82.

12. Witt WP, Cheng ER, Wisk LE, Litzelman K, Chatterjee D, Mandell K. et al. Preterm birth in the United States: the impact of 
stressful life events prior to conception and maternal age. Am J Public Health. 2014;104 Suppl 1:S73-80.

13. Vesterinen HM, Morello-Frosch R, Sen S, Zeise L, Woodruff TJ. Cumulative effects of prenatal-exposure to exogenous chemicals and psychosocial stress on fetal growth: systematic-review of the human and animal evidence. PLoS ONE. 2017;12:e0176331.

14. Morello-Frosch R, Zuk M, Jerrett M, Shamasunder B, Kyle AD. Understanding the cumulative impacts of inequalities in environmental health: implications for policy. Health Aff. 2011;30:879-87.

15. Latendresse $\mathrm{G}$. The interaction between chronic stress and pregnancy: preterm birth from a biobehavioral perspective. J Midwifery Women's Health. 2009;54:8-17.

16. Smith R, Nicholson RC. Corticotrophin releasing hormone and the timing of birth. Front Biosci. 2007;12:912-8.

17. McLean M, Bisits A, Davies J, Woods R, Lowry P, Smith R. A placental clock controlling the length of human pregnancy. Nat Med. 1995;1:460-3.

18. Zhou JJ, Gao Y, Kosten TA, Zhao Z, Li DP. Acute stress diminishes M-current contributing to elevated activity of hypothalamicpituitary-adrenal axis. Neuropharmacology. 2017;114:67-76.

19. Hobel CJ, Dunkel-Schetter C, Roesch SC, Castro LC, Arora CP. Maternal plasma corticotropin-releasing hormone associated with stress at 20 weeks' gestation in pregnancies ending in preterm delivery. Am J Obstet Gynecol. 1999;180:S257-63.

20. Latendresse G, Ruiz RJ. Maternal coping style and perceived adequacy of income predict CRH levels at 14-20 weeks of gestation. Biol Res Nurs. 2010;12:125-36.

21. Mancuso RA, Schetter CD, Rini CM, Roesch SC, Hobel CJ. Maternal prenatal anxiety and corticotropin-releasing hormone associated with timing of delivery. Psychosom Med. 2004;66:762-9.

22. Moog NK, Buss C, Entringer S, Shahbaba B, Gillen DL, Hobel $\mathrm{CJ}$, et al. Maternal exposure to childhood trauma Is associated during pregnancy with placental-fetal stress physiology. Biol Psychiatry. 2016;79:831-9.

23. Wang D-C, Chen T-J, Lin M-L, Jhong Y-C, Chen S-C. Exercise prevents the increased anxiety-like behavior in lactational di-(2ethylhexyl) phthalate-exposed female rats in late adolescence by improving the regulation of hypothalamus-pituitary-adrenal axis. Hormones Behav. 2014;66:674-84.

24. Giesbrecht GF, Liu J, Ejaredar M, Dewey D, Letourneau N, Campbell T, et al. Urinary bisphenol A is associated with dysregulation of HPA-axis function in pregnant women: findings from the APrON cohort study. Environ Res. 2016;151:689-97.

25. Aker AM, Ferguson KK, Rosario ZY, Mukherjee B, Alshawabkeh AN, Calafat AM, et al. A repeated measures study of phenol, paraben and Triclocarban urinary biomarkers and circulating maternal hormones during gestation in the Puerto Rico PROTECT cohort. Environ Health. 2019;18:28.

26. Cathey AL, Watkins D, Rosario ZY, Vélez C, Alshawabkeh AN, Cordero JF, et al. Associations of phthalates and phthalate replacements with $\mathrm{CRH}$ and other hormones among pregnant women in Puerto Rico. J Endocr Soc. 2019;3:1127-49.

27. Ferguson KK, McElrath TF, Meeker JD. Environmental phthalate exposure and preterm birth. JAMA Pediatr. 2014;168:61-7.

28. Mustieles V, Zhang Y, Yland J, Braun JM, Williams PL, Wylie $\mathrm{BJ}$, et al. Maternal and paternal preconception exposure to phenols and preterm birth. Environ Int. 2020;137:105523.

29. Aker A, McConnell RER, Loch-Caruso R, Park SK, Mukherjee B, Rosario $\mathrm{ZY}$, et al. Interactions between chemicals and nonchemical stressors: the modifying effect of life events on the association between triclocarban, phenols and parabens with gestational length in a Puerto Rican cohort. Sci Total Environ. 2020;708:134719.

30. Ferguson KK, Rosen EM, Barrett ES, Nguyen RHN, Bush N, McElrath TF, et al. Joint impact of phthalate exposure and stressful life events in pregnancy on preterm birth. Environ Int. 2019;133:105254.

31. Eick SM, Enright EA, Geiger SD, Dzwilewski KLC, DeMicco E, Smith S, et al. Associations of maternal stress, prenatal exposure to per- and polyfluoroalkyl substances (PFAS), and demographic risk factors with birth outcomes and offspring neurodevelopment: an overview of the ECHO.CA.IL prospective birth cohorts. Int $\mathrm{J}$ Environ Res Public Health. 2021;18:742.

32. Eick SM, Hom Thepaksorn EK, Izano MA, Cushing LJ, Wang Y, Smith SC, et al. Associations between prenatal maternal exposure to per- and polyfluoroalkyl substances (PFAS) and polybrominated diphenyl ethers (PBDEs) and birth outcomes among pregnant women in San Francisco. Environ Health. 2020;19:100.

33. Dohrenwend BS, Krasnoff L, Askenasy AR, Dohrenwend BP. Exemplification of a method for scaling life events: the Peri Life Events Scale. J Health Soc Behav. 1978;19:205-29.

34. Newton RW, Hunt LP. Psychosocial stress in pregnancy and its relation to low birth weight. Br Med $\mathrm{J}$ (Clin Res Ed). 1984;288:1191-4.

35. Kahn JR, Pearlin LI. Financial strain over the life course and health among older adults. J Health Soc Behav. 2006;47:17-31.

36. Anderson S. Core indicators of nutritional state for difficult-tosample populations. J Nutr. 1990;120:1559-600.

37. Blumberg SJ, Bialostosky K, Hamilton WL, Briefel RR. The effectiveness of a short form of the Household Food Security Scale. Am J Public Health. 1999;89:1231-4.

38. Cohen S, Kamarck T, Mermelstein R. A global measure of perceived stress. J Health Soc Behav. 1983;24:385-96.

39. Radloff LS. The CES-D Scale: a self-report depression scale for research in the general population. Appl Psychol Meas. 1977;1:385-401.

40. Eick SM, Goin DE, Izano MA, Cushing L, DeMicco E, Padula AM, et al. Relationships between psychosocial stressors among pregnant women in San Francisco: a path analysis. PLoS ONE. 2020;15:e234579.

41. Cohen S. Perceived stress in a probability sample of the United States. In: The social psychology of health. The Claremont Symposium on Applied Social Psychology. Thousand Oaks, CA, US: Sage Publications, Inc; 1988. p. 31-67.

42. Hall KS, Kusunoki Y, Gatny H, Barber J. The risk of unintended pregnancy among young women with mental health symptoms. Soc Sci Med. 2014;100:62-71.

43. Stidham Hall K, Moreau C, Trussell J, Barber J. Young women's consistency of contraceptive use-does depression or stress matter? Contraception. 2013;88:641-9.

44. Weissman MM, Sholomskas D, Pottenger M, Prusoff BA, Locke BZ. Assessing depressive symptoms in five psychiatric populations: a validation study. Am J Epidemiol. 1977;106:203-14.

45. Sagiv SK, Rifas-Shiman SL, Webster TF, Mora AM, Harris MH, Calafat AM, et al. Sociodemographic and perinatal predictors of early pregnancy per- and polyfluoroalkyl substance (PFAS) concentrations. Environ Sci Technol. 2015;49:11849-58.

46. Chen Y, Holzman C, Chung H, Senagore P, Talge NM, SilerKhodr T. Levels of maternal serum corticotropin-releasing hormone $(\mathrm{CRH})$ at midpregnancy in relation to maternal characteristics. Psychoneuroendocrinology. 2010;35:820-32.

47. Ruiz RJ, Dolbier CL, Fleschler R. The relationships among acculturation, biobehavioral risk, stress, corticotropin-releasing hormone, and poor birth outcomes in Hispanic women. Ethn Dis. 2006; $16: 926-32$.

48. Meltzer-Brody S, Stuebe A, Dole N, Savitz D, Rubinow D, Thorp J. Elevated corticotropin releasing hormone $(\mathrm{CRH})$ during pregnancy and risk of postpartum depression (PPD). J Clin Endocrinol Metab. 2011;96:E40-7.

49. Park SK, Peng Q, Ding N, Mukherjee B, Harlow SD. Determinants of per- and polyfluoroalkyl substances (PFAS) in midlife 
women: Evidence of racial/ethnic and geographic differences in PFAS exposure. Environ Res. 2019;175:186-99.

50. Wadhwa PD, Garite TJ, Porto M, Glynn L, Chicz-DeMet A, Dunkel-Schetter C, et al. Placental corticotropin-releasing hormone $(\mathrm{CRH})$, spontaneous preterm birth, and fetal growth restriction: a prospective investigation. Am J Obstet Gynecol. 2004;191:1063-9.

51. Sandman CA, Wadhwa PD, Chicz-DeMet A, Porto M, Garite TJ. Maternal corticotropin-releasing hormone and habituation in the human fetus. Dev Psychobiol. 1999;34:163-73.

52. Kato K, Wong LY, Chen A, Dunbar C, Webster GM, Lanphear $\mathrm{BP}$, et al. Changes in serum concentrations of maternal poly- and perfluoroalkyl substances over the course of pregnancy and predictors of exposure in a multiethnic cohort of Cincinnati, Ohio pregnant women during 2003-2006. Environ Sci Technol. 2014;48:9600-8.

53. Goin DE, Izano MA, Eick SM, Padula AM, DeMicco E, Woodruff TJ, et al. Maternal experience of multiple hardships and fetal growth: extending environmental mixtures methodology to social exposures. Epidemiology. 2020;32:18-26.

54. Schreier HMC, Hsu H-H, Amarasiriwardena C, Coull BA, Schnaas L, Téllez-Rojo MM, et al. Mercury and psychosocial stress exposure interact to predict maternal diurnal cortisol during pregnancy. Environ Health. 2015;14:28.

55. Rothman KJ. No adjustments are needed for multiple comparisons. Epidemiology. 1990;1:43-6.

56. Nepomnaschy PA, Welch KB, McConnell DS, Low BS, Strassmann BI, England BG. Cortisol levels and very early pregnancy loss in humans. Proc Natl Acad Sci U S A. 2006;103: 3938-42.

57. Bruckner TA, Catalano R, Ahern J. Male fetal loss in the U.S. following the terrorist attacks of September 11, 2001. BMC Public Health. 2010;10:273.

58. Raz R, Kioumourtzoglou MA, Weisskopf MG. Live-birth bias and observed associations between air pollution and autism. Am J Epidemiol. 2018;187:2292-6. 